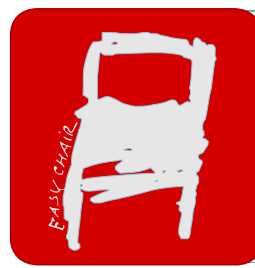

EPiC Series in Health Sciences

CAOS 2018. The 18th Annual Meeting of the International Society for Computer Assisted Orthopaedic Surgery

\title{
Learning Curve in Computer-Assisted Total Knee Arthroplasty: A CUSUM Analysis
}

\author{
Yifei Dai ${ }^{*}$, Guillaume Bras ${ }^{2}$, Cyril Hamad ${ }^{2}$ \\ ${ }^{1 *}$ Exactech Inc, Gainesville, FL, 32653, USA \\ ${ }^{2}$ Blue Ortho, Gieres FR \\ yifei.dai@exac.com
}

\begin{abstract}
This study employed an advanced method (CUSUM) to analyze the learning curve regarding surgical efficiency (time) in 10 surgeons from different experience levels upon their adoption of a contemporary CAOS TKA system. The first 50 CAOS TKA cases of each surgeon were analyzed to identify the case number when their learning process was stabilized (cases of proficiency). The surgical time increase during the learning phase were compared between senior and novice surgeons. The data demonstrated a short learning duration (12-13 cases) and a moderate learning process ( $15 \mathrm{~min}$ time increase compared to later cases) upon adoption of the CAOS system, without high sensitivity to surgeon's experience level.
\end{abstract}

\section{Introduction}

Since the introduction of computer-assisted orthopaedic system (CAOS) in total knee arthroplasty (TKA), the adoption of the system is perceived to be challenged by costs and a significant learning curve. As hospitals are facing mounting financial pressures in the current economic environment, time spent in the operating room has been identified as one of the most costly areas of hospital operations. The successful adoption of a CAOS system should demonstrate superior outcomes compared to conventional TKA, as well as a reasonable learning effort.

To date, limited studies have assessed the learning of new surgical technology or implant system in TKA. The methodology applied in existing studies usually compared surgical time between the cases performed during learning and those from the later cases, with an assumed duration (number of cases) of the learning curve [1-2]. Researchers have performed logarithmic regression on the initial CAOS case series to find the duration of the learning phase [3]. However, as the surgical time data often, by nature, noisy, the regression result can be difficult to evaluate.

Cumulative sum control chart (CUSUM) has been widely applied in the industry to assess the stabilization of a production process, and was proven to be an objective and effective tool to evaluate 
the learning process. Although many successes have been achieved by this method in other medical fields [4], its usage for orthopedic applications, notably TKA research, has been limited. The goal of this study was to leverage this advanced methodology and perform a CUSUM analysis to define the learning period of a contemporary CAOS system.

\section{Materials and Methods}

Technical records on the CAOS TKA cases performed by 10 surgeons (7 senior CAOS surgeons, 3 novice surgeons with no CAOS experience prior to using the system) were reviewed. Surgical time (system usage time) was extracted from each surgeon's first 50 cases. All cases were primary TKA using the Optetrak Logic ${ }^{\circledR}$ Knee System (Exactech Inc, Gainesville, FL, USA) with the assistance of a contemporary CAOS system (ExactechGPS ${ }^{\circledR}$, Blue-Ortho, Gieres, FR).

To perform the CUSUM analysis, the 50 cases from each surgeon were sorted chronologically. The cumulative sum of deviances was calculated as following [4]: The CUSUM of the first case was the difference between the surgical of the first case and the mean surgical time of all 50 cases. The CUSUM of the second case was the previous case's CUSUM plus the difference between the surgical time of the second case and the mean surgical time. This recursive process continued until case \#50, which was calculated as 0. CUSUM value was plotted in chronological order for each surgeon. A horizontal trend in the plot signified the deviances were equally balanced around 0 , indicating the process was operating with stability. The case number (cases to proficiency, abbreviated as CP) by which the CUSUM value entered the horizontal trend was identified as the end of learning curve for each surgeon. The CP was compared between the senior CAOS surgeons and the novice CAOS surgeons. The difference in surgical time was compared between the cases in the learning curve group (from case \#1 to \#CP) and the later cases (\#41-50). Significance was defined as $\mathrm{p}<0.05$.

\section{Results}

Compared to the actual surgical time graph (Fig 1A,C), the CUSUM plot clearly exhibited three unique phases in the first 50 cases of each surgeon, with Phase II demonstrating stabilization of the process (Fig 1B,D). The actual shape of the 3 phases differed from surgeon to surgeon, reflecting each individual's characteristics of learning. On average, it took 12-13 cases to complete the learning curve of the CAOS system assessed, with no substantial difference between the senior surgeon and novice surgeon groups (Table 1). On average both senior and novice surgeon groups spent approximately $15 \mathrm{~min}$ more in surgical time during their learning process than their last 10 cases in the series (\#41-50). The novice surgeons exhibited approximately $3 \mathrm{~min}$ more time increase during their learning phase compared to the senior surgeons. No significant difference was found between senior and novice surgeons regarding $\mathrm{CP}$ and time increase. 

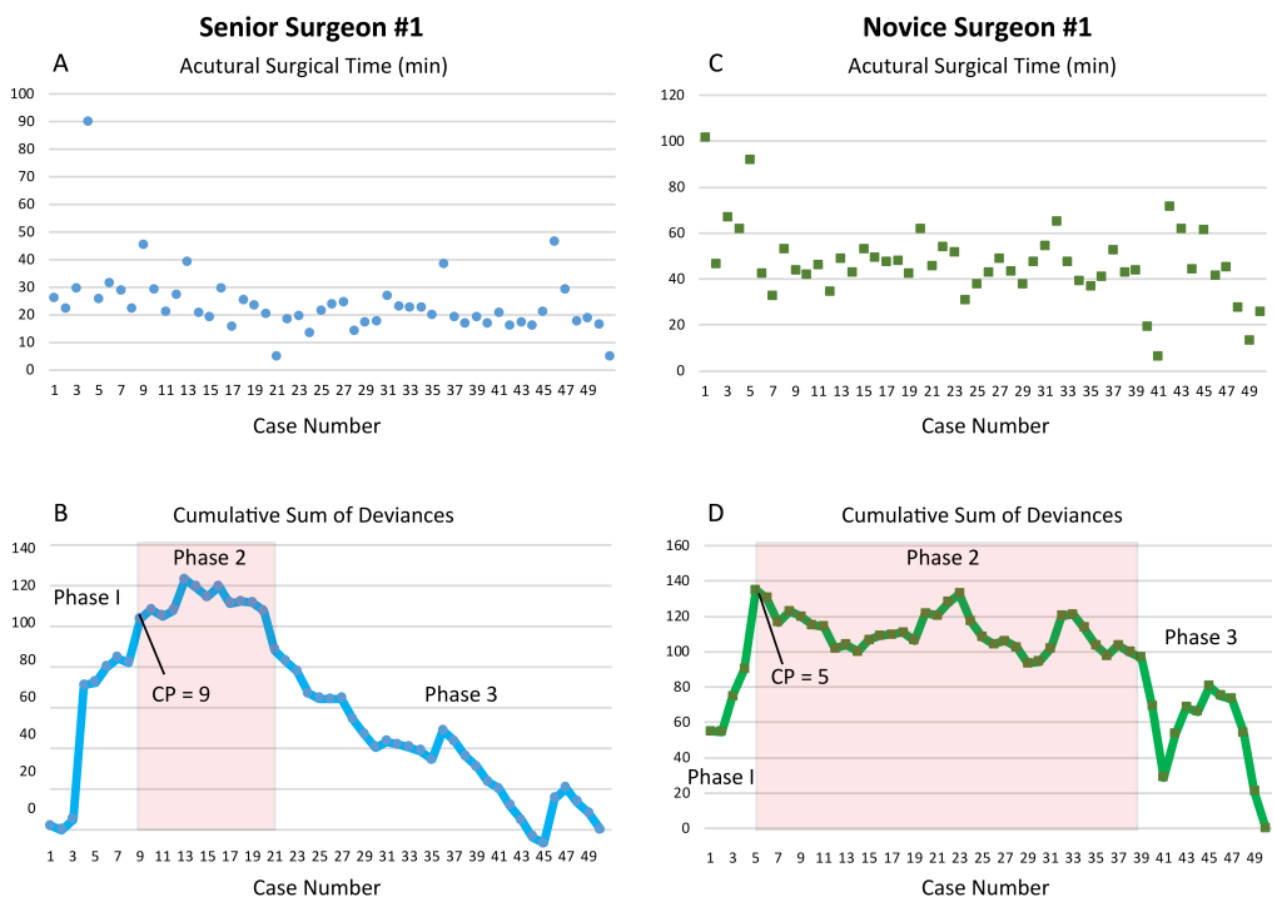

Figure 1. Graphs on the actual surgical time and CUSUM deviance charts for A, B) a representative senior CAOS surgeon, and C, D) a representative novice surgeon. The graph was plotted according to the chronological case numbers.

\begin{tabular}{lccc}
\hline & Senior Surgeon & Novice Surgeon & Pooled \\
$\mathrm{N}$ & 7 & 3 & 10 \\
Cases to Proficiency (CP) & $13.0 \pm 7.1$ & $12.0 \pm 7.5$ & $13.4 \pm 7.4$ \\
Time Increase in Learning Curve $(\mathrm{min})^{*}$ & $13.0 \pm 8.6$ & $16.2 \pm 14.6$ & $13.3 \pm 10.7$ \\
\hline
\end{tabular}

* Calculated as Average(cases \#1 -\#CP) - Average(cases \#41-50)

Table 1. Summary of learning characteristics in the senior surgeons, novice surgeons, and pooled surgeon group. No significant difference was found between senior and novice surgeons (N.S.) in CP and time increase.

\section{Discussion}

This study applied the CUSUM method to analyze the learning curve of a CAOS system for TKA based on surgical efficiency (time), relating the adoption of the surgery as a process that eventually stabilizes with mastery of the task. The data suggested that the average learning of the CAOS system took 12-13 cases, regardless of the surgeon's previous CAOS experience. Compared to cases performed after learning, the learning phase only moderately increased surgical time. For the novice surgeons, the increase of surgical time during the learning phase compared to their later cases did not differ significantly from that of the senior surgeons (16min versus $13 \mathrm{~min}$ ). This indicated that having no CAOS experience before adopting this system did not result in substantially steeper learning curve. 
Learning Curve in Computer-Assisted Total Knee Arthroplasty: A CUSUM Analysis Y. Dai et al.

Despite the CUSUM method has been proposed since the 1970s for analyzing learning curve for surgical procedures [6,7], and since then being applied to various medical fields [4], the use of this method in TKA is very limited [5]. Utilization of this advanced method in studying learning curve not only can provide improved understanding of CAOS learning in general, but also allows differences in learning between individual surgeons or surgeon characteristics to be explored.

\section{References}

[1] Petrera P, Petrera J, Siler X, et al. Exactechgps guidance system does not increase operative time when compared to conventionally instrumented total knee arthroplasty. CAOS 2015

[2] Howard JL, Brenkel I, Chang CB, et al. Learning curve with a new primary tka implant: a worldwide perspective with more than 2000 patients. Bone Joint J 2016.98-B(Supp 20)88.

[3] Jenny JY, Miehlke RK, Giurea A. Learning curve in navigated total knee replacement. A multicentre study comparing experienced and beginner centres. Knee. 2008;15(2):80-4.

[4] Bokhari MB, Patel CB, Ramos-Valadez DI, Ragupathi M, Haas EM. Learning curve for roboticassisted laparoscopic colorectal surgery. Surg Endosc. 2011;25(3):855-60.

[5] De Gori M, Adamczewski B, Jenny JY. Value of the cumulative sum test for the assessment of a learning curve: Application to the introduction of patient-specific instrumentation for total knee arthroplasty in an academic department. Knee. 2017;24(3):615-21.

[6] Chaput de Saintonge DM, Vere DW. Why don't doctors use CUSUMs? Lancet. 1974;1:120-1.

[7] Wohl H. The CUSUM plot: its utility in the analysis of clinical data. N Engl J Med. 1977;296:

1044-5. 